\title{
Dynamics of respiratory symptoms during infancy and associations with wheezing at school age
}

\author{
Jakob Usemann (10 1,2,7, Binbin Xu ${ }^{1,7}$, Edgar Delgado-Eckert ${ }^{1}$, Insa Korten ${ }^{1,2}$, \\ Pinelopi Anagnostopoulou ${ }^{2}$, Olga Gorlanova ${ }^{1}$, Claudia Kuehni $\mathbb{B}^{3}$, \\ Martin Röösli ${ }^{4,5}$, Philipp Latzin $\mathbb{1}^{1,2}$ and Urs Frey, ${ }^{1}$ on behalf of the BILD study \\ group $^{6}$
}

Affiliations: ${ }^{1}$ University Children's Hospital Basel (UKBB), Basel, Switzerland. ${ }^{2}$ Paediatric Respiratory Medicine, Dept of Paediatrics, Inselspital, Bern University Hospital, University of Bern, Bern, Switzerland. ${ }^{3}$ Institute of Social and Preventive Medicine, University of Bern, Bern, Switzerland. ${ }^{4}$ Swiss Tropical and Public Health Institute Basel, Basel, Switzerland. ${ }^{5}$ University of Basel, Basel, Switzerland. ${ }^{6}$ For a list of the members of the BILD study group see the Acknowledgements section. ${ }^{7}$ These authors contributed equally.

Correspondence: Urs Frey, University Children's Hospital Basel, Spitalstrasse 33, 4056 Basel, Switzerland. E-mail: urs.freydukbb.ch

ABSTRACT Children with frequent respiratory symptoms in infancy have an increased risk for later wheezing, but the association with symptom dynamics is unknown. We developed an observerindependent method to characterise symptom dynamics and tested their association with subsequent respiratory morbidity.

In this birth-cohort of healthy neonates, we prospectively assessed weekly respiratory symptoms during infancy, resulting in a time series of 52 symptom scores. For each infant, we calculated the transition probability between two consecutive symptom scores. We used these transition probabilities to construct a Markov matrix, which characterised symptom dynamics quantitatively using an entropy parameter. Using this parameter, we determined phenotypes by hierarchical clustering. We then studied the association between phenotypes and wheezing at 6 years.

In 322 children with complete data for symptom scores during infancy (16864 observations), we identified three dynamic phenotypes. Compared to the low-risk phenotype, the high-risk phenotype, defined by the highest entropy parameter, was associated with an increased risk of wheezing (odds ratio (OR) $3.01,95 \%$ CI $1.15-7.88$ ) at 6 years. In this phenotype, infants were more often male (64\%) and had been exposed to environmental tobacco smoke (31\%). In addition, more infants had siblings (67\%) and attended childcare $(38 \%)$.

We describe a novel method to objectively characterise dynamics of respiratory symptoms in infancy, which helps identify abnormal clinical susceptibility and recovery patterns of infant airways associated with persistent wheezing.

@ERSpublications

Unsupervised analysis of symptom dynamics during infancy identifies subjects susceptible to persistent airway disease http://ow.ly/r9xz30lDpHB

Cite this article as: Usemann J, Xu B, Delgado-Eckert E, et al. Dynamics of respiratory symptoms during infancy and associations with wheezing at school age. ERJ Open Res 2018; 4: 00037-2018 [https://doi.org/10.1183/23120541.00037-2018].

This article has supplementary material available from openres.ersjournals.com

Received: March 052018 | Accepted after revision: Aug 282018

Copyright $\odot$ ERS 2018. This article is open access and distributed under the terms of the Creative Commons Attribution Non-Commercial Licence 4.0. 


\section{Introduction}

Wheezing disorders in early childhood are a major health issue due to their high prevalence [1] and methods to identify infants at risk for subsequent asthma are therefore needed. Exposure to host factors (e.g. sex and maternal atopy) and environmental risk factors (e.g. childcare, siblings, environmental tobacco smoke (ETS) and air pollution) influence the incidence and duration of respiratory symptoms during infancy $[2,3]$, and are associated with wheezing episodes during childhood [4]. In clinical practice, assessing risk factors, estimating the frequency of respiratory symptoms and examining symptom patterns (e.g. episodic versus persistent symptoms) [5] may help identify infants at risk for later asthma.

The pattern of symptom deterioration and recovery may be especially informative, as it is determined by the symptom dynamics that a subject undergoes during a given period of time. While it is known that persistent wheeze in infants is more closely associated with later asthma and reduced lung function than episodic wheeze [5, 6], no previous study has attempted to characterise symptom dynamics in an observer-independent manner, or to estimate their association with persistent respiratory symptoms at school age.

We hypothesise that symptom dynamics are not only determined by exposure to risk factors related to airway infections (e.g. siblings and childcare), but also by host factors and exposure to ETS or air pollution. The symptom dynamics may thus contain information on susceptibility of the airways to infectious triggers. This hypothesis was supported by the previous study of STERN et al. [7], in which infants exposed to higher air pollution levels recovered more slowly from viral infections than those with lower exposure levels.

In order to quantify symptom dynamics, we adapted a Markov matrix approach [8]. Previous studies used a Markov model to study the trajectory of asthma severity [9] and asthma control [10]. We took a similar approach by studying the probabilities of transition between different respiratory symptom states using a Markov matrix and further characterised this matrix using the Shannon entropy [11]. Utilising this measure of disorder, we quantitatively characterised the pattern of symptom deterioration and recovery with one single number.

Using this novel method, our aim was to characterise the symptom dynamics of respiratory symptom scores during infancy, assessed weekly for each infant. We tested whether we could identify a symptom dynamic phenotype of infants with higher risk for persistent wheezing and atopic disease (primary outcomes), and allergic sensitisation, lung function measurements and exhaled nitric oxide fraction ( $F e N O)$ measurements (secondary outcomes) at school age.

Secondly, in order to facilitate the comparison of this study to previous studies $[6,12,13]$, which relied on the total number of infants' symptoms only, we defined similarly sized "reference phenotypes" based on the total number of weeks with respiratory symptoms. We then compared dynamic phenotypes and reference phenotypes relative to the above outcome measures at school age. Lastly, we explored if and how specific dynamic symptom phenotypes are influenced by host factors and environmental risk factors.

\section{Methods}

Study design

In the Basel-Bern Infant Lung Development (BILD) birth-cohort study [14], we prospectively assessed weekly respiratory symptom scores (states 0-4) [15] during infancy, resulting in 52 consecutive observations. We used these symptom scores to construct a Markov matrix for each infant. The entries in this matrix are the empirical probabilities of transitioning from any given symptom score to any other within a week's time. These Markov matrices were further characterised using one single quantitative measure, namely the average Shannon entropy.

We first tested whether we could identify symptom dynamics phenotypes based on this entropy parameter using an unsupervised analysis. We defined similarly sized "reference phenotypes" based on the total number of symptom weeks during infancy. Next, we compared associations between symptom dynamics phenotypes and reference phenotypes with wheezing and atopic disease at 6 years (primary outcomes). Allergic sensitisation, lung function measurements and FeNO measurements were secondary outcomes. Lastly, we compared the distribution of risk factors across phenotypes.

\section{Study participants}

Unselected, healthy term-born neonates were recruited antenatally in Bern and Basel, Switzerland [14]. Between 1999 and 2015, 369 children from Bern were invited for a follow-up. The Ethics Committees of Bern and Basel approved the study. Written informed consent was obtained from parents before enrolment. 


\section{TABLE 1 Respiratory symptom score and associated day and night-time symptoms}

\begin{tabular}{|c|c|c|}
\hline \multirow{2}{*}{$\begin{array}{l}\text { Symptom } \\
\text { score }\end{array}$} & \multicolumn{2}{|c|}{ Symptoms (cough, wheeze, or breathing difficulty) } \\
\hline & Day time & Night time \\
\hline 0 & None & None \\
\hline 1 & Slight; no treatment given & Slight; sleep not disturbed \\
\hline 2 & Required treatment but no outside help & Sleep disturbed once; no help required \\
\hline 3 & Severe; required help from GP & Sleep disturbed more than once or child needed help \\
\hline 4 & Very severe; admitted to hospital & Sleep very disturbed or GP called \\
\hline
\end{tabular}

\section{Exposure: respiratory symptoms during infancy}

During the first year of life, research nurses called the parents weekly to assess respiratory symptoms using standardised symptom scores with four levels of severity (table 1) [3, 15]. "Weeks with any respiratory symptom" were defined as the total number of weeks a child had a respiratory symptom independent of type or severity, while "weeks with severe respiratory symptoms" were defined as a symptom score of three or more (e.g. general practitioner (GP) consultation, as noted previously) [3, 15].

\section{Markov matrices in assessing the dynamics of respiratory symptoms}

We used a Markov model approach to examine dynamic behaviour between consecutive symptom scores. We assessed transitions between different levels of symptom scores ranging from healthy (score 0 ) to most severe (score 4). For each symptom state (i.e. initial state, first time point), we counted how often a transition to any other state (i.e. target state, second time point) occurred, as assessed during the subsequent week. For each infant, this count information was displayed in a $5 \times 5$ matrix (vertical axis=initial state and horizontal axis=target state). These counts were absolute frequencies, which were used to calculate relative frequencies for each transition (figures $1 \mathrm{a}-\mathrm{c}$ ). From this so called Markov matrix [8], we calculated the average Shannon entropy [16] of the empirical conditional probability distributions encoded in each row. Further information on the mathematical approach is provided in figure 2 and the online supplementary material.

\section{Risk factors}

We used a standardised questionnaire to assess pre- and postnatal exposure to risk factors for respiratory symptoms during infancy or asthma development [3]. Parental atopic disease was defined if any of the following was present: self-reported or doctor-diagnosed allergic asthma, history of allergic rhinitis, or atopic dermatitis. Maternal asthma was defined as doctor-diagnosed or self-reported. Maternal education was categorised as low ( 3 years of secondary education) or high ( $\geqslant 4$ years of secondary education). Duration of breastfeeding (exclusive or nonexclusive) was assessed weekly and binary-coded ( $<26$ weeks or $\geqslant 26$ weeks).

\section{Outcomes: respiratory and allergic diseases at 6 years}

Asthma and allergy were assessed by an adapted International Study of Asthma and Allergies in Childhood (ISAAC) questionnaire. We chose the outcomes "any wheezing" (wheezing between one and 6 years) and "current wheezing" (wheezing over the past 12 months before follow-up). Atopic disease was defined as allergic rhinitis, allergic asthma, or atopic dermatitis. Allergic sensitisation was assessed using a skin-prick test (SPT), determined to be positive if a wheal diameter in any of the tested aeroallergens was greater than in a positive control [14]. Spirometry was performed according to guidelines [17]. Forced expiratory volume in $1 \mathrm{~s}$ (FEV1), forced vital capacity (FVC), FEV1/FVC ratio and forced expiratory flow at $25-75 \%$ of FVC (FEF25-75\%) were expressed as z-scores [18]. We measured online FeNO according to current guidelines [19].

\section{Statistical analysis}

Entropy is a measure of disorder within a dynamic system and higher entropy values correspond to more irregularity [11]. The higher the fluctuation between many different respiratory symptoms, the higher is the entropy. For each infant, we calculated the average Shannon entropy [20] over the rows of the Markov matrix, which provided a quantitative measure of the irregularity patterns of symptom deterioration and recovery. Ward's hierarchical clustering [21] was performed to identify phenotypes based on the entropy. 
a)

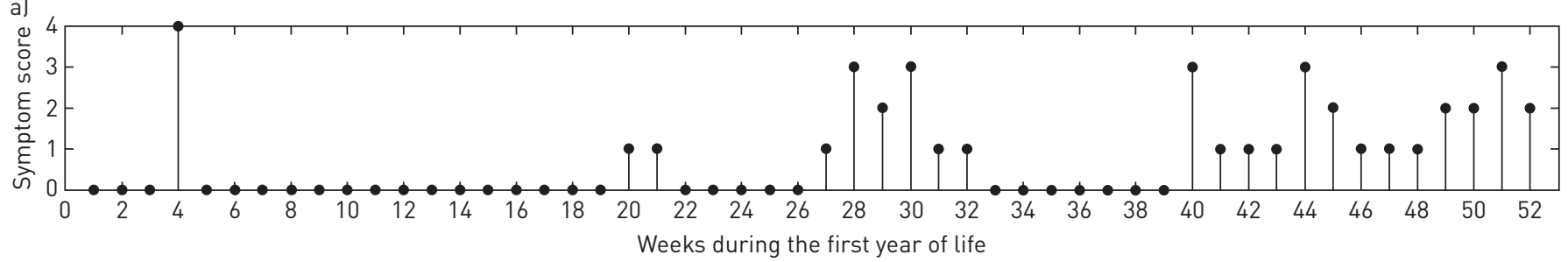

b)

Markov matrix written in terms of the absolute numbers of transitions observed

\begin{tabular}{|c|c|c|c|c|c|}
\hline 4 & 1 & 0 & 0 & 0 & 0 \\
\hline 3 & 0 & 2 & 3 & 0 & 0 \\
\hline 2 & 0 & 1 & 1 & 2 & 0 \\
\hline 1 & 2 & 6 & 1 & 2 & 0 \\
\hline \multirow[t]{2}{*}{0} & 26 & 2 & 0 & 1 & 1 \\
\hline & 0 & 1 & 2 & 3 & 4 \\
\hline
\end{tabular}

Target state $0-4$ c)

Markov matrix written in terms of the relative frequencies (in percentage) of transitions observed

\begin{tabular}{|c|c|c|c|c|c|}
\hline 4 & 100 & 0 & 0 & 0 & 0 \\
\hline 3 & 0 & 40 & 60 & 0 & 0 \\
\hline 2 & 0 & 25 & 25 & 50 & 0 \\
\hline 1 & 18.2 & 54.5 & 9.1 & 18.2 & 0 \\
\hline 0 & 86.7 & 6.7 & 0 & 3.3 & 3.3 \\
\hline & 0 & 1 & 2 & 3 & 4 \\
\hline
\end{tabular}

Target state $0-4$

FIGURE 1 Distribution of respiratory symptom states during infancy of one infant and corresponding Markov matrices. Panel (a) shows the Temporal pattern of respiratory symptom scores during the first year of life for one infant. We assessed transitions between different levels of the symptom scores (state 0: healthy state, symptom score 0; states 1-4: symptomatic states, symptom scores 1-4). For each symptom state (i.e. initial state, 1st time point), we counted how often a transition to any other state (i.e. target state, 2nd time point) occurred, as assessed during the subsequent week. This count information is displayed in a $5 \times 5$ matrix. Absolute values are displayed in panel (b), whereas the corresponding relative frequencies are displayed in panel (c).

The optimal number of clusters and thereby of phenotypes was determined by the majority of indices [22]. We defined similarly sized "reference phenotypes" by the frequency distribution of the total number of weeks with respiratory symptoms during infancy. Using logistic regression, we studied the association of symptom dynamic phenotypes, reference phenotypes and asthma risk factors with outcomes. For the outcomes any wheezing, current wheezing, atopic disease and positive SPT, analyses were adjusted for sex, maternal education, maternal asthma, ETS exposure, childcare, and siblings. FeNO was additionally adjusted for hay fever and inhaled corticosteroid (ICS) use. For lung function, we adjusted for maternal education, maternal asthma, ETS exposure, childcare and siblings [23]. We used Chi-squared and Kruskal-Wallis tests to compare characteristics across phenotypes and accounted for multiple testing. We used the weighted kappa-statistic to compare agreement between symptom dynamic phenotypes and reference phenotypes.

For sensitivity analyses, we repeated the analysis in infants who had one or more episodes with a symptom score of three or more and also within an additional, independent sample from our cohort of 242 infants. To explore whether the entropy distribution was an artefact of our analysis, we re-categorised the symptom states, simulated data and perturbed the existing data. Furthermore, we assessed the robustness of our findings after correcting for unobserved events. In particular, we theoretically explored the repercussions of observing more severe respiratory symptoms.

\section{Results}

From 369 eligible subjects, 322 (87\%) were studied (see supplementary figure E1), each having $\geqslant 50$ weeks of symptom series during infancy and complete data on risk factors and outcomes (table 2). Demographic data and distribution of respiratory symptoms did not differ between infants followed-up and those lost to follow-up. With respect to risk factors, infants lost to follow-up were more frequently exposed to ETS and were less breastfed (see supplementary table E1).

\section{Distribution of respiratory symptoms}

In infants followed-up, we had information for 16864 person-weeks. The number of weeks with any respiratory symptom was determined (on average) as median 4 weeks (range 0-23 weeks). In contrast, severe symptoms were rare at median 0 weeks (range $0-6$ weeks). The distribution of all symptom states is shown in figure 3. 

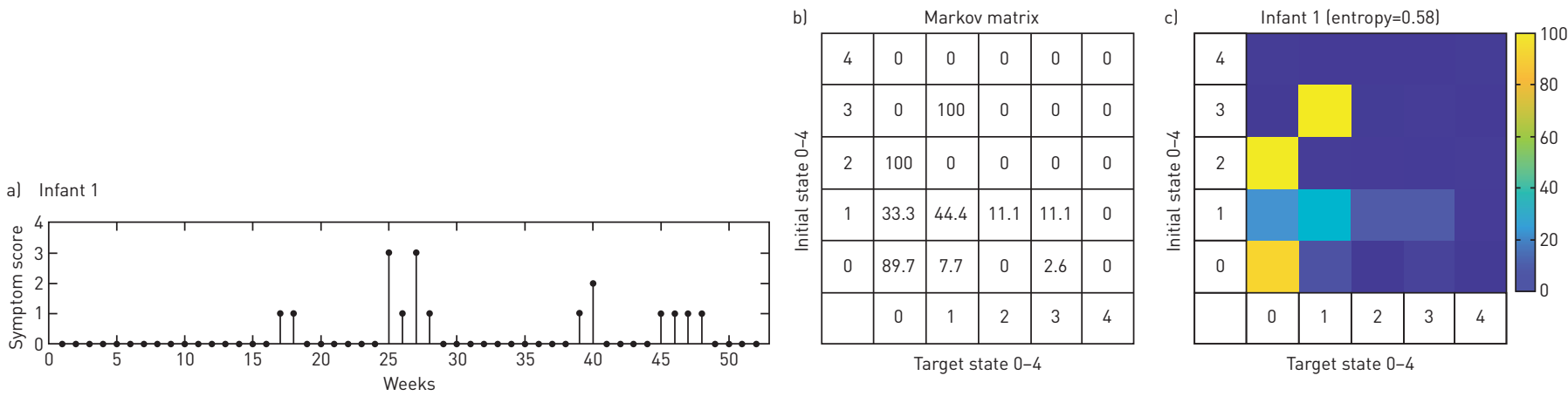

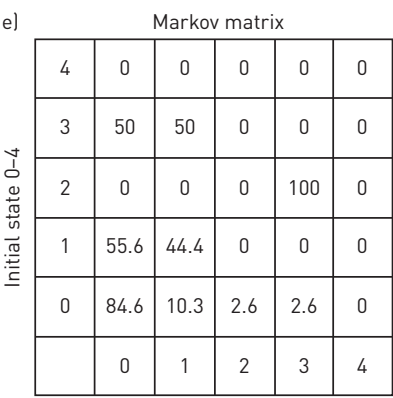

Target state $0-4$

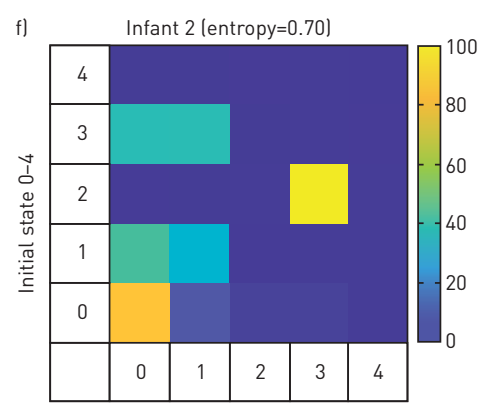

Target state $0-4$

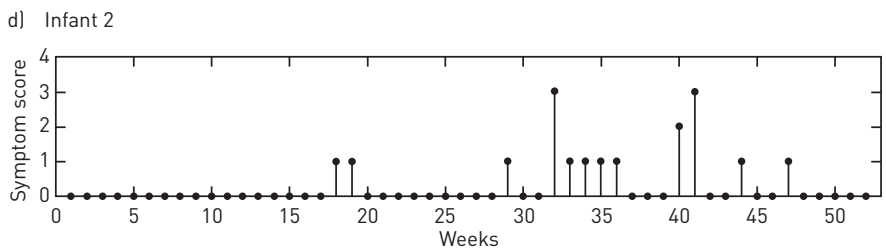

FIGURE 2 Representative Markov matrix patterns of two infants. Panel (a) (infant 1) and panel (d) (infant 2) show the weekly respiratory symptom scores (states 0-4) during the first year of life. The total number of "weeks with respiratory symptoms" during the first year for both infants were the same (37 weeks symptom score 0,9 weeks symptom score 1, 1 week symptom score 2 and 2 weeks symptom score 3 ). The Markov matrices in panel (b) (infant 1) and panel (e) (infant 2) show the empirical probability of each transition (described in the methods and figure 1). In panels (c) and ( $f$ ), the colours correspond to the empirical probability of each transition. The pattern of the Markov matrix landscape was expressed using a single average entropy parameter [16] (described in the supplementary material), which was 0.58 for infant 1 and 0.70 for infant 2.

\section{Dynamics of respiratory symptoms assessed by the Markov matrix}

The dynamic patterns of respiratory symptoms for two infants are shown in figure 2. Both had the same number of symptom weeks and the same distribution of symptom scores, but different patterns for the Markov matrix landscape. The shape of the landscape as characterised by a single entropy parameter differed as well. Infant 1 (figures $2 \mathrm{a}-\mathrm{c}$ ) had an entropy of 0.58 versus infant 2 (figures $2 \mathrm{~d}-\mathrm{f}$ ) who had an entropy of 0.70 . The higher entropy value for infant 2 indicates higher fluctuation between respiratory symptom scores compared to infant 1 .

\section{Outcomes at 6 years}

From 322 children followed-up, 105 (32.9\%) had an outcome of any wheezing and $38(11.7 \%)$ had an outcome of current wheezing. There were 120 children (37.5\%) with atopic disease and a subgroup of 270 completed a SPT, of which $37(13.6 \%)$ were positive (table 2). Lung function tests were completed in 222 children (68.7\%) and 231 (71.7\%) had FeNO measurements.

\section{Identification of phenotypes}

Hierarchical Ward's clustering was feasible and identified three symptom dynamic phenotypes. We defined three similarly sized reference phenotypes using the frequency distribution of the total number of weeks with respiratory symptoms.

\section{Over- and under-representation of risk factors across phenotypes Symptom dynamic phenotypes}

The three symptom dynamic phenotypes included 145, 135 and 42 infants, respectively (table 3). They differed with regard to the number of weeks with any symptoms and severe symptoms (phenotype 1 had severe symptoms for 0.22 weeks, phenotype 2 for 0.82 weeks and phenotype 3 for 2.11 weeks). Entropy also differed across phenotypes (phenotype 1 had an entropy of 0.08 , phenotype 2 of 0.56 and phenotype 3 of 1.03) and so did risk factors. Phenotype 1 had an under-representation of infants with siblings and with childcare attendance, and infants were more likely to be born via Caesarean section. Compared to phenotype 1, phenotype 2 had more infants with siblings and with childcare attendance, and infants were less exposed to ETS. In phenotype 3, the smallest group, more infants had siblings and attended childcare, 
TABLE 2 Characteristics of the study population

\begin{tabular}{|c|c|c|c|c|}
\hline & n $(\%)$ & Mean士sd & Median (IQR) & Range \\
\hline \multicolumn{5}{|l|}{ Anthropometric data at birth } \\
\hline Gestational age weeks & & $39.6 \pm 1.2$ & $39.8(38.8-40.5)$ & $36.7-41.8$ \\
\hline Birth weight kg & & $3.3 \pm 0.4$ & $3.3(3.0-3.6)$ & $2.1-4.9$ \\
\hline Length $\mathrm{cm}$ & & $49.5 \pm 1.9$ & $50.0(48-51)$ & $44-57$ \\
\hline \multicolumn{5}{|c|}{ Respiratory symptoms in the first year of life } \\
\hline Weeks with symptoms & & $5.3 \pm 4.6$ & $4(0-20)$ & $0-23$ \\
\hline Weeks with severe symptoms & & $0.71 \pm 1.1$ & $0(0-1)$ & $0-6$ \\
\hline \multicolumn{5}{|l|}{ Risk factors } \\
\hline Male sex & $167(51.8)$ & & & \\
\hline Siblings & $159(49.4)$ & & & \\
\hline Caesarean section & $54(16.7)$ & & & \\
\hline Maternal asthma & 34 (10.5) & & & \\
\hline Maternal atopy & $116(36.1)$ & & & \\
\hline Childcare & 62 (19.5) & & & \\
\hline Maternal smoking in pregnancy & $27(8.4)$ & & & \\
\hline Parental smoking during infancy & 70 (21.7) & & & \\
\hline Breastfeeding $>26$ weeks & 252 (78.3) & & & \\
\hline Low maternal education & $202(62.7)$ & & & \\
\hline \multicolumn{5}{|l|}{ Season of birth } \\
\hline Spring & $87(27.4)$ & & & \\
\hline Summer & $83(25.7)$ & & & \\
\hline Autumn & 80 (24.8) & & & \\
\hline Winter & $72(22.6)$ & & & \\
\hline \multicolumn{5}{|l|}{ Outcomes at 6 year follow-up } \\
\hline Any wheezing ${ }^{\#}$ & 105 (32.9) & & & \\
\hline Current wheezing & $38(11.7)$ & & & \\
\hline Atopic disease $^{+}$ & $120(37.5)$ & & & \\
\hline SPT positive ${ }^{\S}$ & $37(13.6)$ & & & \\
\hline
\end{tabular}

Data are derived from 322 infants with a total of 16864 observed symptom weeks. IQR: interquartile range; SPT: skin-prick test. \#: defined as any wheezing episode between one and 6 years of age; ${ }^{7}$ : defined as wheezing over the past 12 months before follow-up; ${ }^{+}$: defined as allergic rhinitis, allergic asthma, or atopic dermatitis before follow-up; ${ }^{\S}$ : a SPT was completed in a subset of 270 children.

and slightly more infants were male and were exposed to ETS. We considered phenotype 3 a high-risk symptom dynamic phenotype since associations with both later wheezing and atopic disease were strongest.

\section{Reference phenotypes}

The three phenotypes included 147, 128 and 47 infants, respectively (table 3). Phenotypes 1, 2 and 3 had $0.14,0.87$ and 2.14 weeks with severe symptoms, respectively. Entropy was 0.13 for phenotype 1, 0.54 for phenotype 2 and 0.91 for phenotype 3. Risk factors differed across phenotypes. Phenotype 1 had an under-representation of males and fewer infants had siblings. Infants were also more likely to be born via Caesarean section. In phenotype 2, infants were less likely to be born via Caesarean section and in phenotype 3 there was an over-representation of males and more infants had siblings.

\section{Association of symptom dynamic phenotypes, reference phenotypes and risk factors with outcomes at 6 years}

In the adjusted logistic regression model, male gender and maternal asthma were significantly associated with any wheezing during childhood. Male gender was also associated with current wheezing, any wheezing and atopic disease. Symptom dynamic phenotype 3 was significantly associated with any wheezing and current wheezing, compared to symptom dynamic phenotype 1 , whereas symptom dynamic phenotype 2 was not associated with these outcomes. Reference phenotypes 2 and 3 were significantly associated with any wheezing compared to reference phenotype 1 (table 4). There was a significant association between both maternal asthma and symptom dynamic phenotype 3 toward wheezing between 2 and 3 years of age, whereas reference phenotypes 2 and 3 were not associated with this outcome (see supplementary table E2). None of the phenotypes were associated with the outcome positive SPT (see supplementary table E3). For sensitivity analysis, we repeated the entire analysis and obtained similar 


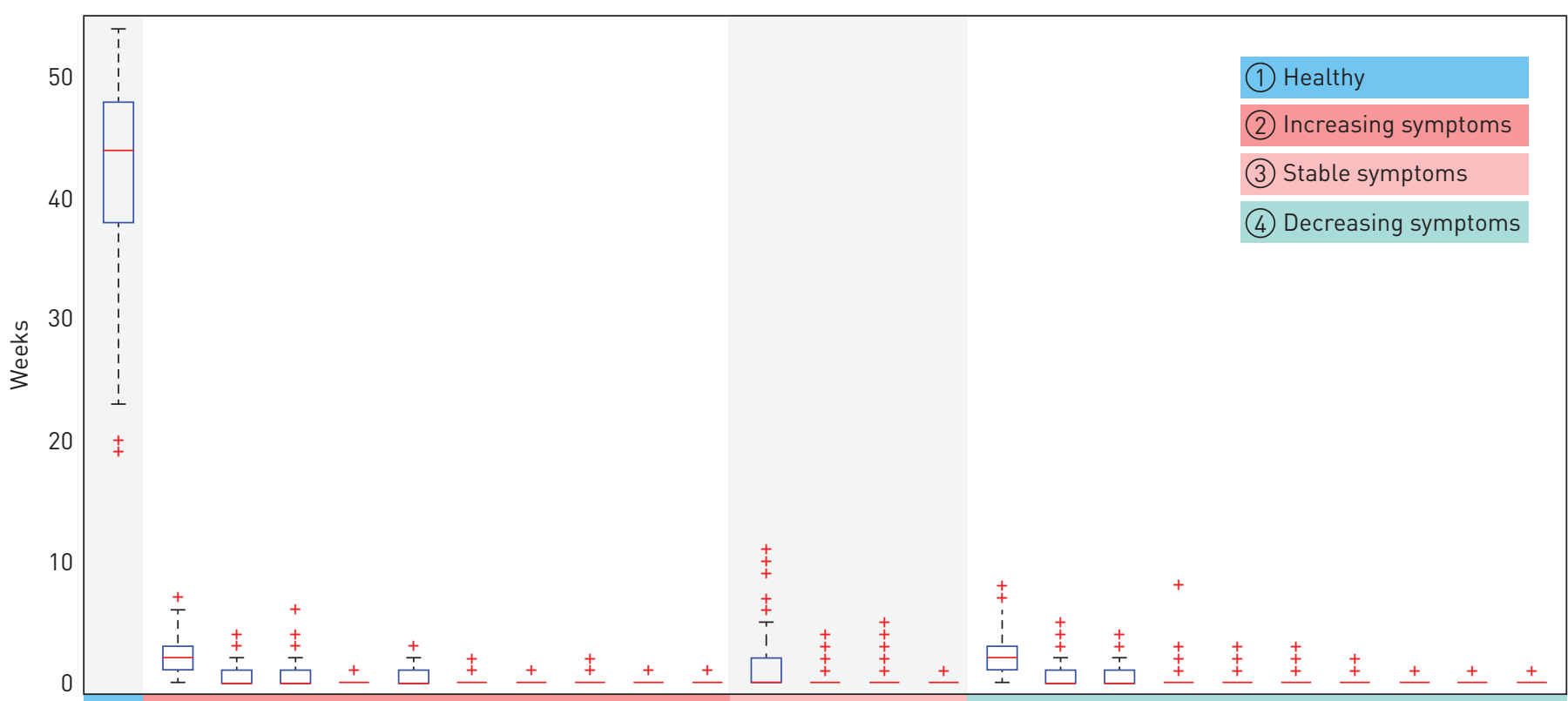

$(0-0)(0-1)(0-2)(0-3)(0-4)(1-2)(1-3)(1-4)(2-3)(2-4)(3-4)(1-1)(2-2)(3-3)(4-4)(1-0)(2-0)(2-1)(3-0)(3-1)(3-2)(4-0)(4-1)(4-2)(4-3)$ (1) (2)

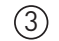

Transitions
(4)

FIGURE 3 Respiratory symptom transitions of all study participants. The vertical-axis represents the number of transitions (in weeks) observed during the first year of life. The horizontal-axis represents all possible transitions (Type 1: healthy state; Type 2: increasing symptoms; Type 3: stable symptoms; Type 4: decreasing symptoms). Data are shown as box plots, while the numbers in brackets indicate initial symptom score (initial state) and target symptom score (target state), as assessed in the subsequent week.

results in infants with one or more episodes with a symptom score of three or more (data not shown). There was no association between any of the predictors and lung function and FeNO measurements at 6 years (see supplementary table E4).

\section{Comparison between symptom dynamic phenotypes and reference phenotypes}

There was a high degree of overlap between the dynamic and reference phenotypes, with a weighted kappa-statistic value of 0.61 . The overlap of infants allocated to each phenotype was 107 (74\%) to phenotype $1,76(56 \%)$ to phenotype 2 and $20(48 \%)$ to phenotype 3 . Infants in dynamic phenotype 3 had greater fluctuation between respiratory symptom scores compared to reference phenotype 3 (entropy 1.03 versus 0.91 ). This indicates that phenotyping based on symptom dynamics identifies infants with different characteristics compared to the reference method based on the number of symptom weeks alone. For example, in high-risk symptom dynamic phenotype 3, we found an over-representation of infants from smoking parents; whereas, in reference phenotype 3, other factors (e.g. maternal atopy) were over-represented (table 3).

\section{Sensitivity analyses}

The robustness of our findings was assessed in various sensitivity analyses. These are discussed in the supplementary material.

\section{Discussion}

We developed a novel method, which, based on a time series of weekly symptom scores from healthy infants in the first year of life, characterised symptom dynamics (i.e. the pattern of symptom deterioration and recovery) in an observer-independent fashion. With this method we identified three symptom dynamic phenotypes, of which one had more infants attending childcare and a greater number of infants with siblings. In addition, the number of male infants slightly higher and there was more exposure to ETS. Compared to the reference phenotype, this dynamic phenotype had a slightly higher prevalence for wheeze (26\% versus $21 \%)$ and atopic disease $(46 \%$ versus $36 \%)$ at 6 years. Of all clinically measured exposures in infancy in our unselected cohort, infants in this dynamic phenotype had the highest OR for wheeze between one and 6 years (OR 4.31, 95\% CI 1.95-9.48) and current wheeze at 6 years (OR 3.01, 95\% CI 1.15-7.88). These findings suggest that host factors and susceptibility to environmental factors in 
TABLE 3 Characteristics of symptom dynamic phenotypes and reference phenotypes

\begin{tabular}{|c|c|c|c|c|c|c|c|c|}
\hline & \multicolumn{2}{|c|}{ Phenotype 1} & \multicolumn{2}{|c|}{ Phenotype 2} & \multicolumn{2}{|c|}{ Phenotype 3} & \multicolumn{2}{|c|}{ p-value } \\
\hline & $\begin{array}{l}\text { Dynamic } \\
\text { ( } n=145 \text { ) }\end{array}$ & $\begin{array}{l}\text { Reference } \\
(n=147)\end{array}$ & $\begin{array}{l}\text { Dynamic } \\
(n=135)\end{array}$ & $\begin{array}{c}\text { Reference } \\
(n=128)\end{array}$ & $\begin{array}{c}\text { Dynamic } \\
(\mathrm{n}=42)\end{array}$ & $\begin{array}{c}\text { Reference } \\
\quad(n=47)\end{array}$ & Dynamic & Reference \\
\hline \multicolumn{9}{|l|}{ Respiratory symptoms } \\
\hline Weeks with severe symptoms & $0.22 \pm 0.43$ & $0.14 \pm 0.37$ & $0.82 \pm 1.03$ & $0.87 \pm 0.98$ & $2.11 \pm 1.64$ & $2.14 \pm 1.53$ & $<0.001$ & $<0.001$ \\
\hline Weeks with any symptoms & $1.84 \pm 1.58$ & $1.57 \pm 1.09$ & $7.03 \pm 3.64$ & $6.61 \pm 1.96$ & $12.19 \pm 3.56$ & $13.87 \pm 3.03$ & $<0.001$ & $<0.001$ \\
\hline \multicolumn{9}{|l|}{ Respiratory symptoms transition states } \\
\hline Entropy of transition states & $0.08 \pm 0.05$ & $0.13 \pm 0.16$ & $0.56 \pm 0.14$ & $0.54 \pm 0.25$ & $1.03 \pm 0.12$ & $0.91 \pm 0.23$ & $<0.001$ & $<0.001$ \\
\hline \multicolumn{9}{|l|}{ Risk factors } \\
\hline Male sex & 50.3 & 43.5 & 49.6 & 56.3 & 64.3 & 65.9 & 0.223 & 0.012 \\
\hline Siblings & 37.2 & 38.1 & 57.1 & 53.9 & 66.7 & 72.4 & $<0.001$ & $<0.001$ \\
\hline Caesarean section & 24.8 & 23.8 & 10.4 & 10.2 & 9.5 & 12.7 & 0.002 & 0.008 \\
\hline Maternal asthma & 9.6 & 8.8 & 9.6 & 10.2 & 16.7 & 17.1 & 0.385 & 0.278 \\
\hline Maternal atopy & 37.2 & 33.3 & 34.8 & 35.9 & 35.7 & 44.7 & 0.914 & 0.370 \\
\hline Childcare & 13.7 & 11.5 & 19.3 & 20.3 & 38.1 & 40.4 & 0.006 & $<0.001$ \\
\hline Maternal smoking during pregnancy & 7.6 & 6.8 & 8.2 & 10.2 & 11.9 & 8.5 & 0.668 & 0.606 \\
\hline Parental smoking during infancy & 24.8 & 23.1 & 15.5 & 20.3 & 30.9 & 21.3 & 0.051 & 0.805 \\
\hline Breastfeeding $\leqslant 26$ weeks & 21.4 & 20.4 & 21.5 & 23.4 & 23.8 & 21.3 & 0.941 & 0.829 \\
\hline Low maternal education & 64.1 & 61.9 & 62.2 & 64.1 & 59.5 & 61.7 & 0.851 & 0.983 \\
\hline \multicolumn{9}{|l|}{ Season of birth } \\
\hline Spring & 25.5 & 26.5 & 31.1 & 27.3 & 19.1 & 27.7 & 0.264 & 0.983 \\
\hline Summer & 22.1 & 18.4 & 27.4 & 35.9 & 33.3 & 21.3 & 0.289 & 0.003 \\
\hline Autumn & 35.8 & 35.4 & 16.3 & 15.6 & 14.3 & 17.1 & $<0.001$ & $<0.001$ \\
\hline Winter & 16.5 & 19.7 & 25.2 & 21.1 & 33.3 & 34.1 & 0.042 & 0.111 \\
\hline \multicolumn{9}{|l|}{ Outcomes at 6 year follow-up } \\
\hline Any wheezing ${ }^{\#}$ & 27.6 & 22.4 & 28.9 & 38.3 & 64.3 & 51.1 & $<0.001$ & $<0.001$ \\
\hline Current wheezing? & 9.6 & 8.8 & 9.6 & 11.7 & 26.2 & 21.3 & 0.008 & 0.071 \\
\hline Atopic disease $^{+}$ & 35.4 & 36.3 & 37.1 & 39.4 & 46.3 & 36.2 & 0.449 & 0.855 \\
\hline SPT positive ${ }^{\S}$ & 13.3 & 14.5 & 12.3 & 13.1 & 19.4 & 13.2 & 0.545 & 0.942 \\
\hline \multicolumn{9}{|c|}{$\begin{array}{l}\text { Data are presented as } \% \text { or mean } \pm \text { SD. Symptom dynamic phenotypes were defined by the average entropy of transition states and reference phenotypes by weeks with any respiratory } \\
\text { symptom. Differences in the distribution of characteristics across phenotypes were assessed using Chi-squared tests for categorical variables and Kruskal-Wallis tests for continuous } \\
\text { variables. Significant } p \text {-values at the Bonferroni-corrected } \alpha \text {-level of } 0.017 \text { are shown in bold. SPT: skin-prick test. "\# : defined as any wheezing episode between one and } 6 \text { years of age; } \\
{ }^{\prime} \text { : defined as wheezing over the past } 12 \text { months before follow-up; }{ }^{+} \text {: defined as allergic rhinitis, allergic asthma, or atopic dermatitis before follow-up; }{ }^{\S} \text { : a SPT was completed in a subset } \\
\text { of } 270 \text { children. }\end{array}$} \\
\hline
\end{tabular}




\begin{tabular}{|c|c|c|c|c|c|c|}
\hline \multirow[t]{2}{*}{ Outcome } & \multicolumn{3}{|c|}{ Univariable association } & \multicolumn{3}{|c|}{ Multivariable association" } \\
\hline & OR & $95 \% \mathrm{Cl}$ & p-value & OR & $95 \% \mathrm{Cl}$ & p-value \\
\hline \multicolumn{7}{|c|}{ Any wheezing between one and 6 years ${ }^{\Uparrow}(n=105 / 322)$} \\
\hline \multicolumn{7}{|c|}{ Reference phenotypes } \\
\hline Phenotype 1 (baseline; $\mathrm{n}=147$ ) & 1 & Reference & & 1 & Reference & \\
\hline Phenotype $2(n=128)$ & 2.14 & $1.26-3.62$ & 0.005 & 1.93 & $1.11-3.35$ & 0.019 \\
\hline Phenotype $3(n=47)$ & 3.61 & $1.81-7.19$ & $<0.001$ & 2.84 & $1.32-6.11$ & 0.008 \\
\hline \multicolumn{7}{|l|}{ Symptom dynamic phenotypes } \\
\hline Phenotype 1 (baseline; $n=145$ ) & 1 & Reference & & 1 & Reference & \\
\hline Phenotype 2 ( $n=135$ ) & 1.07 & $0.63-1.79$ & 0.809 & 1.06 & $0.61-1.84$ & 0.830 \\
\hline Phenotype 3 ( $n=42$ ) & 4.72 & $2.27-9.72$ & $<0.001$ & 4.31 & $1.95-9.48$ & $<0.001$ \\
\hline \multicolumn{7}{|l|}{ Risk factors } \\
\hline Male sex & 3.05 & $1.86-5.01$ & $<0.001$ & 2.90 & $1.75-4.81$ & $<0.001$ \\
\hline Siblings & 1.22 & $0.77-1.95$ & 0.386 & 1.27 & $0.78-2.09$ & 0.328 \\
\hline Maternal asthma & 2.23 & $1.09-4.58$ & 0.028 & 2.16 & $1.03-4.55$ & 0.041 \\
\hline Childcare & 1.49 & $0.84-2.64$ & 0.169 & 1.40 & $0.75-2.59$ & 0.284 \\
\hline Parental smoking during infancy & 1.08 & $0.61-1.89$ & 0.783 & 0.99 & $0.55-1.81$ & 0.995 \\
\hline Low maternal education & 1.23 & $0.76-2.01$ & 0.391 & 1.13 & $0.66-1.93$ & 0.644 \\
\hline \multicolumn{7}{|l|}{ Current wheezing at 6 years $^{+}(n=38 / 322)$} \\
\hline \multicolumn{7}{|l|}{ Reference phenotypes } \\
\hline Phenotype 1 (baseline; n=147) & 1 & Reference & & 1 & Reference & \\
\hline Phenotype $2(n=128)$ & 1.36 & $0.62-2.99$ & 0.433 & 1.22 & $0.54-2.75$ & 0.618 \\
\hline Phenotype $3(n=47)$ & 2.78 & $1.13-6.86$ & 0.026 & 2.25 & $0.83-6.11$ & 0.110 \\
\hline \multicolumn{7}{|l|}{ Symptom dynamic phenotypes } \\
\hline Phenotype 1 (baseline; $n=145$ ) & 1 & Reference & & 1 & Reference & \\
\hline Phenotype 2 ( $n=135$ ) & 0.99 & $0.45-2.21$ & 0.994 & 1.01 & $0.44-2.31$ & 0.968 \\
\hline Phenotype 3 (n=42) & 3.32 & $1.37-8.01$ & 0.008 & 3.01 & $1.15-7.88$ & 0.025 \\
\hline \multicolumn{7}{|l|}{ Risk factors } \\
\hline Male sex & 2.52 & $1.21-5.28$ & 0.014 & 2.26 & $1.06-4.81$ & 0.033 \\
\hline Siblings & 1.15 & $0.58-2.28$ & 0.670 & 1.19 & $0.61-2.42$ & 0.616 \\
\hline Maternal asthma & 1.71 & $0.65-4.45$ & 0.141 & 1.66 & $0.62-4.41$ & 0.306 \\
\hline Childcare & 1.35 & $0.61-3.02$ & 0.462 & 1.38 & $0.58-3.27$ & 0.457 \\
\hline Parental smoking during infancy & 1.33 & $0.61-2.89$ & 0.468 & 1.15 & $0.51-2.59$ & 0.723 \\
\hline Low maternal education & 1.77 & $0.82-3.78$ & 0.141 & 1.63 & $0.73-3.66$ & 0.229 \\
\hline \multicolumn{7}{|l|}{ Atopic disease at 6 years $^{\S}(n=120 / 320)$} \\
\hline \multicolumn{7}{|l|}{ Reference phenotypes } \\
\hline Phenotype 1 (baseline; $n=146$ ) & 1 & Reference & & 1 & Reference & \\
\hline Phenotype 2 ( $n=127)$ & 1.13 & $0.69-1.86$ & 0.602 & 1.06 & $0.63-1.78$ & 0.824 \\
\hline Phenotype $3(n=47)$ & 0.99 & $0.51-1.97$ & 0.987 & 0.89 & $0.41-1.91$ & 0.772 \\
\hline \multicolumn{7}{|l|}{ Symptom dynamic phenotypes } \\
\hline Phenotype 1 (baseline; $n=144$ ) & 1 & Reference & & 1 & Reference & \\
\hline Phenotype 2 ( $n=135$ ) & 1.07 & $0.65-1.74$ & 0.778 & 1.12 & $0.66-1.88$ & 0.667 \\
\hline Phenotype $3(n=41)$ & 1.57 & $0.78-3.17$ & 0.205 & 1.74 & $0.81-3.77$ & 0.159 \\
\hline \multicolumn{7}{|l|}{ Risk factors } \\
\hline Male sex & 2.15 & $1.35-3.42$ & 0.001 & 2.10 & $1.29-3.40$ & 0.003 \\
\hline Siblings & 1.09 & $0.69-1.72$ & 0.686 & 1.01 & $0.63-1.63$ & 0.944 \\
\hline Maternal asthma & 1.55 & $0.76-3.18$ & 0.226 & 1.51 & $0.72-3.17$ & 0.274 \\
\hline Childcare & 0.62 & $0.34-1.14$ & 0.127 & 0.63 & $0.33-1.21$ & 0.168 \\
\hline Parental smoking during infancy & 1.09 & $0.63-1.88$ & 0.752 & 0.92 & $0.55-1.64$ & 0.786 \\
\hline Low maternal education & 2.30 & $1.40-3.77$ & 0.001 & 2.03 & $1.20-3.41$ & 0.008 \\
\hline \multicolumn{7}{|c|}{$\begin{array}{l}\text { Logistic regression analysis was carried out for the outcomes any wheezing, current wheezing and atopic } \\
\text { disease. Symptom dynamic phenotypes were defined by average entropy of transition states and reference } \\
\text { phenotypes by weeks with any respiratory symptom. When considering phenotypes as exposure, phenotype } \\
1 \text { from the reference phenotype or symptom dynamic phenotype, respectively, served as baseline. OR: } \\
\text { odds ratio. " } \text { : adjusted for the binary variables male gender, low maternal education, maternal asthma, } \\
\text { maternal smoking during pregnancy, childcare attendance during infancy and presence of siblings; } \\
\text { १: defined as any wheezing episode between one and } 6 \text { years of age; }{ }^{+} \text {: defined as wheezing over the past } \\
12 \text { months before follow-up; }{ }^{\S} \text { : defined as allergic rhinitis, allergic asthma, or atopic dermatitis before } \\
\text { follow-up. }\end{array}$} \\
\hline
\end{tabular}


infancy are not only associated with the total number of symptoms, but are associated with symptom dynamics even more so. The same is even truer if we consider the subsequent persistence of airway symptoms into childhood. Although we observed an expected overlap between the symptom dynamic and reference phenotypes, our findings are consistent with the hypothesis that symptom dynamics provide relevant information on airway susceptibility and recovery patterns. The latter may be relevant for our understanding of airway vulnerability in the development of chronic disease.

\section{Comparison with the literature}

Previous studies using unsupervised methods assessed symptoms at two timepoints [24] and up to 14 timepoints [12] during childhood. The resolution of respiratory symptoms in our study is unique, since we had a minimum of 50 observations per infant. Comparison of phenotypes between this study and previous studies is limited given that previous studies phenotyped based on "wheeze" during childhood [6, 13, 24-26], while our study focused on "any respiratory symptom" during infancy. Previous studies often investigated the association of phenotypes with risk factors during childhood (e.g. allergic sensitisation), while our study specifically assessed prenatal (e.g. maternal smoking during pregnancy) and early postnatal risk factors (e.g. breastfeeding and childcare attendance). The symptom dynamics and total number of symptom weeks during infancy were not associated with lung function or FeNO at 6 years. These findings differ from the results in a large European birth cohort (the ALSPAC study) describing an association between different wheezing phenotypes (e.g. early transient and persistent wheeze) and lung function at 8-9 years $[6,12]$. Differences could be due to the small number of lung function measurements performed at 6 years in our study, or that only a few children had severely reduced lung function in this unselected study population. Furthermore, while our method assessed "any respiratory symptom" for phenotyping (mostly cough and rhinitis), the ALSPAC study assessed "wheezing" $[6,12]$, which may reflect other airway properties.

\section{Potential mechanisms, and interplay between host and environmental risk factors}

Interplay between an exposure (e.g. virus or pollutants) and host predisposition (e.g. infant from asthmatic mothers or male sex) is hypothesised to determine response to a respiratory infection. Studies further suggest that the severity of respiratory infections in children depends on virus type [27], microbial composition [28] and environmental factors [3,29]. The interplay between these factors is suggested to affect airway epithelial function [30, 31]. Further prospective studies may assess if previously suggested markers to assess epithelial function (e.g. cytokine expression and impaired interferon response) [30] are relevant for future disease.

\section{Strengths and limitations}

We prospectively assessed respiratory symptoms weekly by telephone interview, obtaining reliable data on respiratory morbidity [15] and reducing potential recall bias compared to retrospectively reported symptoms. We had few missing data points, with only two infants out of all eligible participants being excluded for having less than 50 weeks of observations. We used the same questionnaire to assess risk factors and outcome data throughout the entire study period at both centres, reducing potential inter-centre differences. Since this study was conducted in an unselected population, there were only 38 subjects with current wheezing at 6 years. However, the high-risk phenotypes incorporated only small numbers of infants and as such misclassification of even one subject could majorly influence our findings. While the number of participants lost to follow-up was low (13\%), a potential selection bias may still have influenced our findings. Few cohort studies prospectively assessed respiratory symptoms during infancy $[3,13,32]$. Due to a lack of data, we could not validate our method in any of those studies, but did perform an external validation in 242 infants from our cohort not yet seen for follow-up (see supplementary table E5). We performed several sensitivity analyses to rule out that the distribution of entropy was a methodological artefact. For example, we tested whether this distribution could be a mathematical artefact related to the scoring system (five states). However, recategorisation of the states $(0,1,2,(3+4) \rightarrow 3)$, did not systematically change the identified phenotypes. After correcting for unobserved events, we investigated the scenario of analysing a hypothetical population at higher risk. This analysis resulted in higher entropy values and also a different entropy distribution (see supplementary material). These results suggest that the phenotypes obtained using our method may depend on the distribution of symptom severity. Consequently, an external validation of our method in a different study population may yield different phenotypes.

\section{Clinical relevance and research applications}

Our results indicate that specific symptom dynamics during infancy are associated with wheezing at 6 years. The developed method could potentially be used in ongoing research on asthma control and risk prediction, as it objectively quantifies symptom patterns and could be considered complementary to routine asthma biomarkers (e.g. asthma predictive index, FeNO and lung function). Furthermore, 
assessment of symptom patterns is noninvasive and does not require laboratory equipment. It could thus be used in primary care and telemonitoring settings [33]. Smartphone applications for the assessment of symptoms are already available and have been used in patients with asthma [34], cystic fibrosis [35], and rhinitis [36]. These novel tools may enable automatic, low-cost assessment of symptoms from which especially high-risk populations may benefit. The mathematical algorithm could be integrated with the tools' software, which would enable the calculation of symptom dynamics without profound expertise, as well as in larger patient groups.

\section{Conclusion}

In this study, we developed a method to quantitatively characterise the symptom dynamics of prospectively assessed respiratory symptoms and found that specific dynamic symptom patterns were associated with host and environmental factors and persistent wheezing up to 6 years in a high-risk phenotype. Although host factors (male sex) and environmental factors (ETS exposure during infancy) were more frequent in this phenotype, these infants were predominantly exposed to infectious risk factors (siblings and childcare) in the first year. Beyond solely looking at the total number of symptoms, the dynamics of symptom patterns may provide relevant information on the susceptibility and recovery capacity of the airways after environmental stimuli in infancy. The developed method has potential in various research settings and might contribute to our understanding of airway susceptibility associated with persistent airway disease.

Acknowledgements: The authors thank the parents for participating in the study, and the study nurses Christine Becher Monika Graf and Linda Beul-Béguin (Paediatric Respiratory Medicine, Dept of Paediatrics, Inselspital, Bern University Hospital, University of Bern, Bern, Switzerland) for performing the weekly phone calls to the parents, and the entire BILD cohort team. We would also like to thank Karine Hugentobler (University Children's Hospital Basel (UKBB), Basel, Switzerland) for her critical reading of the manuscript.

Author contributions: J. Usemann, P. Latzin, M. Röösli, C. Kuehni and U. Frey were investigators in this study and contributed to the study design. I. Korten, A. Schmidt, P. Anagnostopoulou, E. Proietti, O. Fuchs, O. Gorlanova and J. Usemann are members of the BILD study team and contributed to data collection and analysis. J. Usemann, B. Xu, M. Röösli, C. Kuehni, P. Latzin, E. Delgado-Eckert and U. Frey contributed to the data interpretation. E. Delgado-Eckert and $\mathrm{B}$. Xu conceived the computational approach. All authors contributed to the writing and review of the report, and approved the final version of the manuscript.

The current Basel-Bern Infant Lung Development (BILD) cohort study group is: Pinelopi Anagnostopoulou, Bern Urs Frey, Basel; Oliver Fuchs, Bern; Olga Gorlanova, Basel; Insa Korten, Bern; Claudia Kuehni, Bern; Philipp Latzin, Bern; Elena Proietti, Zurich; Martin Röösli, Basel; Anne Schmidt, London; and Jakob Usemann, Basel.

Conflict of interest: P. Latzin reports receiving personal fees from Vertex, Novartis, Roche, Polyphor, Vifor and Gilead outside the submitted work.

Support statement: This study was supported by grants from the Swiss National Science Foundation (grant 320030_163311 awarded to U. Frey, P. Latzin and C. Kuehni, and grant 32003B_162820 awarded to C. Kuehni) and a research fellowship from the Austrian, German and Swiss Paediatric Respiratory Society (awarded to J. Usemann). Funding information for this article has been deposited with the Crossref Funder Registry.

\section{References}

$1 \quad$ Braman SS. The global burden of asthma. Chest 2006; 130: 4S-12S

2 Gorlanova O, Thalmann S, Proietti E, et al. Effects of breastfeeding on respiratory symptoms in infancy. J Pediatr 2016; 174: 111-117.

3 Latzin P, Frey U, Roiha HL, et al. Prospectively assessed incidence, severity, and determinants of respiratory symptoms in the first year of life. Pediatr Pulmonol 2007; 42: 41-50.

4 Morgan WJ, Stern DA, Sherrill DL, et al. Outcome of asthma and wheezing in the first 6 years of life: follow-up through adolescence. Am J Respir Crit Care Med 2005; 172: 1253-1258.

5 Frey U, von Mutius E. The challenge of managing wheezing in infants. N Engl J Med 2009; 360: 2130-2133.

6 Henderson J, Granell R, Heron J, et al. Associations of wheezing phenotypes in the first 6 years of life with atopy, lung function and airway responsiveness in mid-childhood. Thorax 2008; 63: 974-980.

7 Stern G, Latzin P, Roosli M, et al. A prospective study of the impact of air pollution on respiratory symptoms and infections in infants. Am J Respir Crit Care Med 2013; 187: 1341-1348.

8 Asmussen S. Applied probability and queues. 2nd Edn. New York, Springer, 2003.

9 Chen W, Marra CA, Lynd LD, et al. The natural history of severe asthma and influences of early risk factors: a population-based cohort study. Thorax 2016; 71: 267-275.

10 Combescure C, Chanez P, Saint-Pierre P, et al. Assessment of variations in control of asthma over time. Eur Respir J 2003; 22: 298-304.

11 Bishop CM. Pattern recognition and machine learning. New York, Springer, 2006.

12 Granell R, Henderson AJ, Sterne JA. Associations of wheezing phenotypes with late asthma outcomes in the Avon Longitudinal Study of Parents and Children: a population-based birth cohort. J Allergy Clin Immunol 2016; 138: 1060-1070.

13 Depner M, Fuchs O, Genuneit J, et al. Clinical and epidemiologic phenotypes of childhood asthma. Am J Respir Crit Care Med 2014; 189: 129-138. 
14 Fuchs O, Latzin P, Kuehni CE, et al. Cohort profile: the Bern infant lung development cohort. Int J Epidemiol 2012; 41: 366-376.

15 Silverman M, Wang M, Hunter G, et al. Episodic viral wheeze in preschool children: effect of topical nasal corticosteroid prophylaxis. Thorax 2003; 58: 431-434.

16 Pratt WK. Chapter 15: Image feature extraction. In: Introduction to digital image processing. Boca Raton, CRC Press, 2013.

17 Miller MR, Hankinson J, Brusasco V, et al. Standardisation of spirometry. Eur Respir J 2005; 26: 319-338.

18 Quanjer PH, Stanojevic S, Cole TJ, et al. Multi-ethnic reference values for spirometry for the 3-95-yr age range: the global lung function 2012 equations. Eur Respir J 2012; 40: 1324-1343.

19 American Thoracic Society, European Respiratory Society. ATS/ERS recommendations for standardized procedures for the online and offline measurement of exhaled lower respiratory nitric oxide and nasal nitric oxide, 2005. Am J Respir Crit Care Med 2005; 171: 912-930.

20 Paninski L. Estimation of entropy and mutual information. Neural Computation 2003; 15: 1191-1253.

21 Ward JH, Jr. Hierarchical grouping to optimize an objective function. J Am Stat Assoc 1963; 58: 236-244.

22 Charrad M, Ghazzali N, Boiteau V, et al. NbClust: an R package for determining the relevant number of clusters in a data set. J Stat Softw 2014; 61: 1-36.

23 Dogaru CM, Strippoli MP, Spycher BD, et al. Breastfeeding and lung function at school age: does maternal asthma modify the effect? Am J Respir Crit Care Med 2012; 185: 874-880.

24 Spycher BD, Silverman M, Brooke AM, et al. Distinguishing phenotypes of childhood wheeze and cough using latent class analysis. Eur Respir J 2008; 31: 974-981.

25 Garcia-Aymerich J, Benet M, Saeys Y, et al. Phenotyping asthma, rhinitis and eczema in MeDALL population-based birth cohorts: an allergic comorbidity cluster. Allergy 2015; 70: 973-984

26 Spycher BD, Silverman M, Pescatore AM, et al. Comparison of phenotypes of childhood wheeze and cough in 2 independent cohorts. J Allergy Clin Immunol 2013; 132: 1058-1067.

27 Carlsson CJ, Vissing NH, Sevelsted A, et al. Duration of wheezy episodes in early childhood is independent of the microbial trigger. J Allergy Clin Immunol 2015; 136: 1208-1214.

28 von Mutius E. The microbial environment and its influence on asthma prevention in early life. J Allergy Clin Immunol 2016; 137: 680-689.

29 Caudri D, Savenije OE, Smit HA, et al. Perinatal risk factors for wheezing phenotypes in the first 8 years of life. Clin Exp Allergy 2013; 43: 1395-1405.

30 Cook J, Saglani S. Pathogenesis and prevention strategies of severe asthma exacerbations in children. Curr Opin Pulm Med 2016; 22: 25-31.

31 Holt PG, Sly PD. Viral infections and atopy in asthma pathogenesis: new rationales for asthma prevention and treatment. Nat Med 2012; 18: 726-735.

32 Bonnelykke K, Vissing NH, Sevelsted A, et al. Association between respiratory infections in early life and later asthma is independent of virus type. J Allergy Clin Immunol 2015; 136: 81-86.

33 de Jongste JC, Carraro S, Hop WC, et al. Daily telemonitoring of exhaled nitric oxide and symptoms in the treatment of childhood asthma. Am J Respir Crit Care Med 2009; 179: 93-97.

34 Cook KA, Modena BD, Simon RA. Improvement in asthma control using a minimally burdensome and proactive smartphone application. J Allergy Clin Immunol Pract 2016; 4: 730-737.

35 Wood J, Jenkins S, Putrino D, et al. High usability of a smartphone application for reporting symptoms in adults with cystic fibrosis. J Telemed Telecare 2018; 24: 547-552.

36 Caimmi D, Baiz N, Tanno LK, et al. Validation of the MASK-rhinitis visual analogue scale on smartphone screens to assess allergic rhinitis control. Clin Exp Allergy 2017; 47: 1526-1533. 